\title{
Techno-economic Analysis of a Dynamic Impairment-Aware Optical Network
}

\author{
D. Staessens ${ }^{1}$, M. Angelou ${ }^{2}$, M. De Groote ${ }^{1}$, S. Azodolmolky ${ }^{2}$, D. Klonidis ${ }^{2}$, \\ S.Verbrugge ${ }^{1}$, D. Colle ${ }^{1}$, M. Pickavet ${ }^{1}$ and I. Tomkos ${ }^{2}$ \\ ${ }^{1}$ Ghent University - IBBT, Gaston Crommenlaan 8 bus 201, 9050 Ghent, Belgium \\ ${ }^{2}$ Athens Information Technology, 19.5 km, Markopoulo Ave., 19002, Peania, Athens, Greece \\ dimitri.staessens@intec.ugent.be,mang@ait.edu.gr
}

\begin{abstract}
We quantified the performance of impairment-aware networking in terms of capital and operational expenditures and compared it to an impairment-unaware solution in the presence of nodes that bear different degrees of flexibility.

(C) 2010 Optical Society of America

OCIS codes: (060.4510) Optical communications; (060.6718) Switching, circuit
\end{abstract}

\section{Introduction}

When planning or upgrading a long haul core network it is important to optimize investments by balancing the cost and frequency of equipment installations. Previous work has shown the economic benefits of transparent networking, with Capital Expenditures (CapEx) savings up to $50 \%$ by avoiding unnecessary optical-electronic-optical (O/E/O) conversions [1] and significant Operational Expenditures (Opex) savings due to the minimal power consumption of photonics [2]. However in a transparent network as the physical-layer impairments accumulate, the quality of transmission (QoT) degrades and therefore error free detection of the optical signal can be achieved only for a finite distance [3]. In this context Impairment Aware (IA) transparent networks make maximum use of the optical reach as this leads to lower blocking rates and more efficient utilization of resources.

In addition to the photonic switches that effectively enable transparency, reconfigurable optical switching components are adopted due to their flexibility to support dynamic traffic in a cost-effective manner [4]. Reconfigurable optical networks allow operators not to over-provision their network with equipment meant to serve future variations in traffic. In this work we study CapEx and OpEx reductions achieved by an IA networking solution under the flexibility constraints of different node architecture and show that the IA solution achieves better resource optimization when compared to an Impairment Unaware (IUA) solution without QoT considerations.

\section{Methodology}

To assess the IA network planning, we dimension a reference network, comprising of 14 nodes and 23 bidirectional links, using the offline IA Routing and Wavelength Assignment (IA-RWA) algorithm proposed in [5]. The IA-RWA algorithm is compared to an IUA method that does not consider any physical-layer constraints. The two algorithms are fed with the same traffic matrices and their output (essentially the lightpaths that serve the traffic demands) is used to calculate the capital and operational costs, by adding the required component costs (amplifiers, add/drop terminals, transponders, network interfaces) of each solution. The IUA-RWA algorithm that was employed stems from the IARWA algorithm, yet only taking into account the wavelength availability and continuity constraints. The output of the IUA algorithm is checked afterwards for feasibility by the same QoT estimator used by the IA-RWA algorithm. To quantify the planning solutions of the two RWA approaches we assume that the network is equipped with 80-channel line systems. If the 80-channel system is exhausted in terms of resources, a parallel line system is installed.

Additionally, the analysis is performed for four (4) different types of node architectures that differ in terms of flexibility (bearing or not the features: directionless, colorless, contentionless). The cost of the different architectures depends on their physical implementation, presented in detail in [6]. The CapEx and OpEx model for the various node components and the capabilities of the four node architectures are summarized in Table 1. It is based on [6] and updated through a survey of the most recent list prices published by component manufacturers and system vendors [7]. 
Table 1: Component CapEx, Network-related Opex and node capabilities [7]

\begin{tabular}{|c|c|c|c|}
\hline Equipment & Relative cost & Power consumption [W] & Mean Time Between Failures \\
\hline Long reach $10 \mathrm{G}$ Transponder & 1 & 30 & 250,000 \\
\hline EDFA, double stage & 1.33 & 25 & 500,000 \\
\hline EDFA, single stage & 1 & 15 & 500,000 \\
\hline 1x4 WSS (80 channels) & 2.35 & 30 & 300,000 \\
\hline $1 \times 8$ WSS & 4.7 & 40 & 300,000 \\
\hline $1 \times 20$ WSS & 7.05 & 50 & 300,000 \\
\hline 1x40 WSS (not commercially available) & 10.58 & 60 & 150,000 \\
\hline Splitter(cost per port) & 0.05 & - & - \\
\hline Combiner(cost per port) & 0.13 & - & - \\
\hline AWG & 0.7 & - & - \\
\hline Casing Node & 2.5 & - & - \\
\hline Casing Network Interface & $1-4$ & $2-8$ & - \\
\hline Casing AWG & 0.5 & - & - \\
\hline Floorspace (Cost per square meter per year) & 0.27 & - & \\
\hline Power (Cost per kW per year) & 0.79 & - & - \\
\hline Repair cost per WSS failure & 0.2 & - & - \\
\hline Repair cost per Transponder failure & 0.1 & - & - \\
\hline Repair cost EDFA double/single stage & 0.1 & - & - \\
\hline Node type & Directionless & Colorless & Contentionless \\
\hline R-OXC6 & no & no & yes \\
\hline $\mathrm{R}-\mathrm{OXC} 4$ & no & yes & no \\
\hline $\mathrm{R}-\mathrm{OXC} 3$ & yes & yes & no \\
\hline $\mathrm{R}-\mathrm{OXC} 2$ & yes & yes & partly \\
\hline
\end{tabular}

\section{Results}

We consider a traffic load scaling from 0.8 to 1.1 , where load 1 corresponds to a traffic matrix with one 10-Gb/s connection request per pair of nodes (i.e. 182 lightpaths for the 14-node reference network). The CapEx results with respect to the induced traffic load for R-OXC6 are depicted in Fig. 1 (left). For load 0.8, the two methods yield the same CapEx as both can serve the traffic with the 80 available channels. As the load increases, the cost of the IA network solution rises slightly due to the number of transponders, related to the increased number of demands, but it can still serve all of the demands on a single 80 channel line system. The IUA algorithm, however, requires more than 80 channels to accommodate the traffic, leading to a significant increase in CapEx. To resolve the physical blocking an extra parallel line system is installed, which translates to a cost increase of around $60 \%$ when compared to the IA counterpart. Fig. 1 clearly shows that the IA solution outperforms the IUA one in CapEx and the respective difference is directly proportional to the extra equipment that is required to serve the traffic without any blocking. The IUA solution does not consider the QoT in the lightpath computation and therefore the routing and channel allocation is not optimized. As a consequence, the IUA approach calls for a capital investment sooner than the IA one as the traffic increases. The two methods were also assessed in terms of CapEx assuming R-OXC4 (Fig. 1 right). The overall higher computed cost for R-OXC4 when compared to R-OXC6 is due to the increased flexibility of the node.

Fig. 2 illustrates the relative cost for the four architectures for load 0.8 (left) and load 1.1 (right). The cost is driven primarily by the number of add/drop terminals and the network interfaces. As a consequence, regardless of the traffic load the most flexible node, i.e. R-OXC2, appears as the most costly. In the OpEx estimations, the repair costs
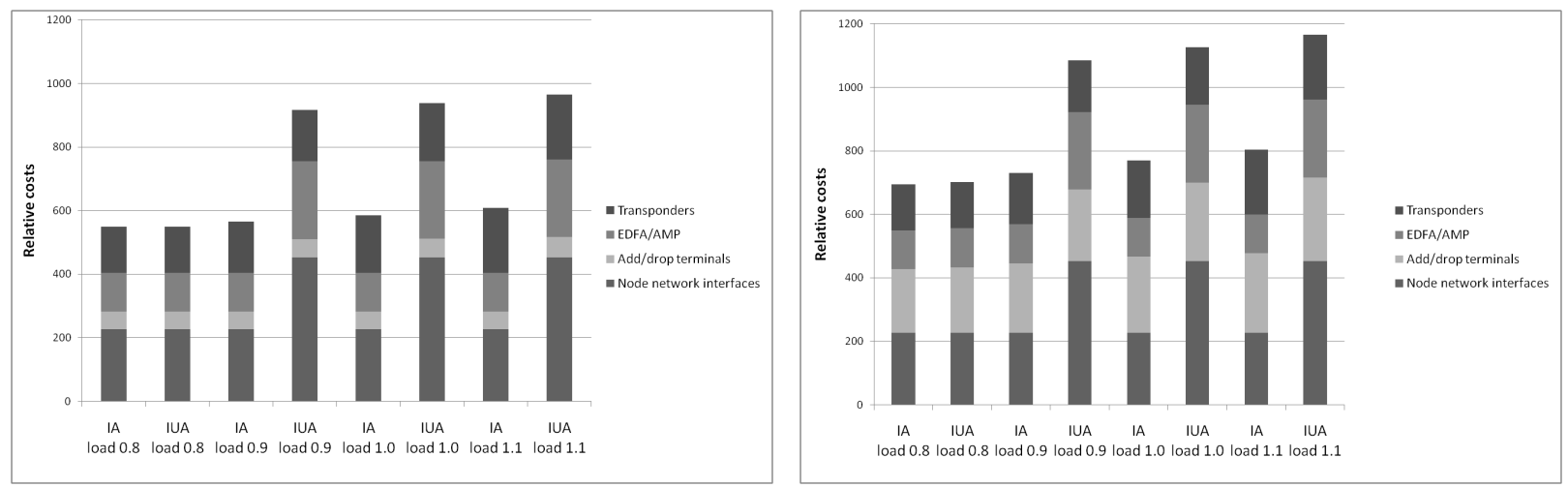

Fig. 1: CapEx of IA vs. IUA for R-OXC6 (left); CapEx of IA vs. IUA for R-OXC4 (right). 

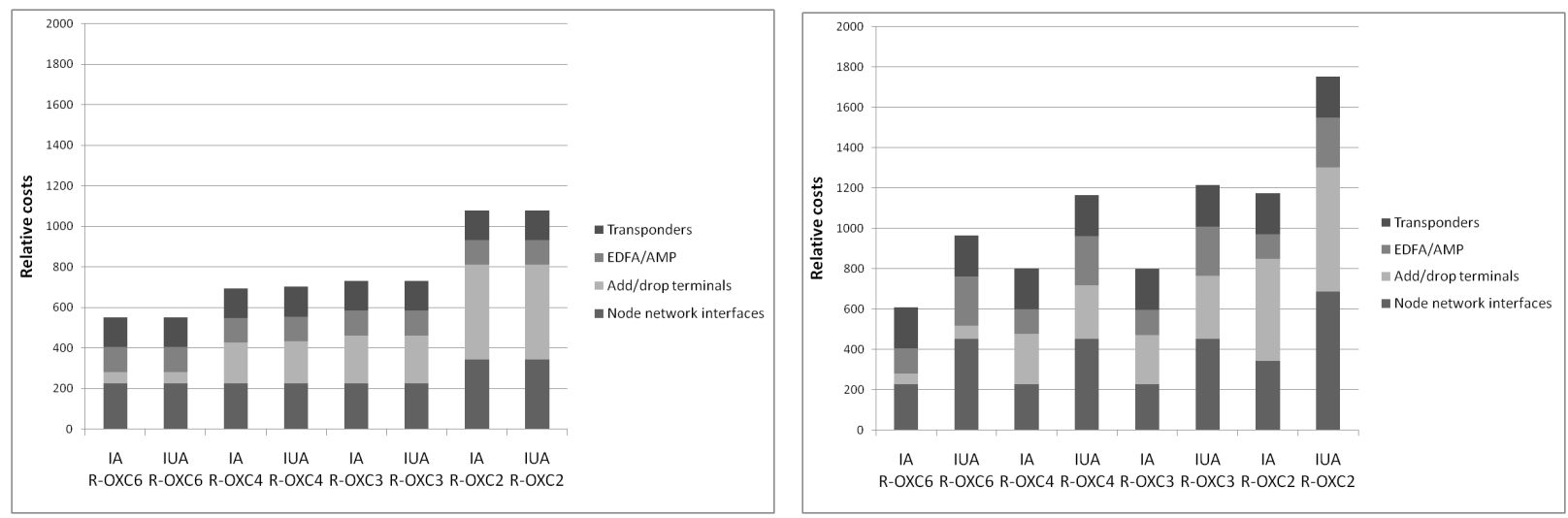

Fig. 2: CapEx of the different node architectures for load 0.8 (left) and load 1.1 (right).
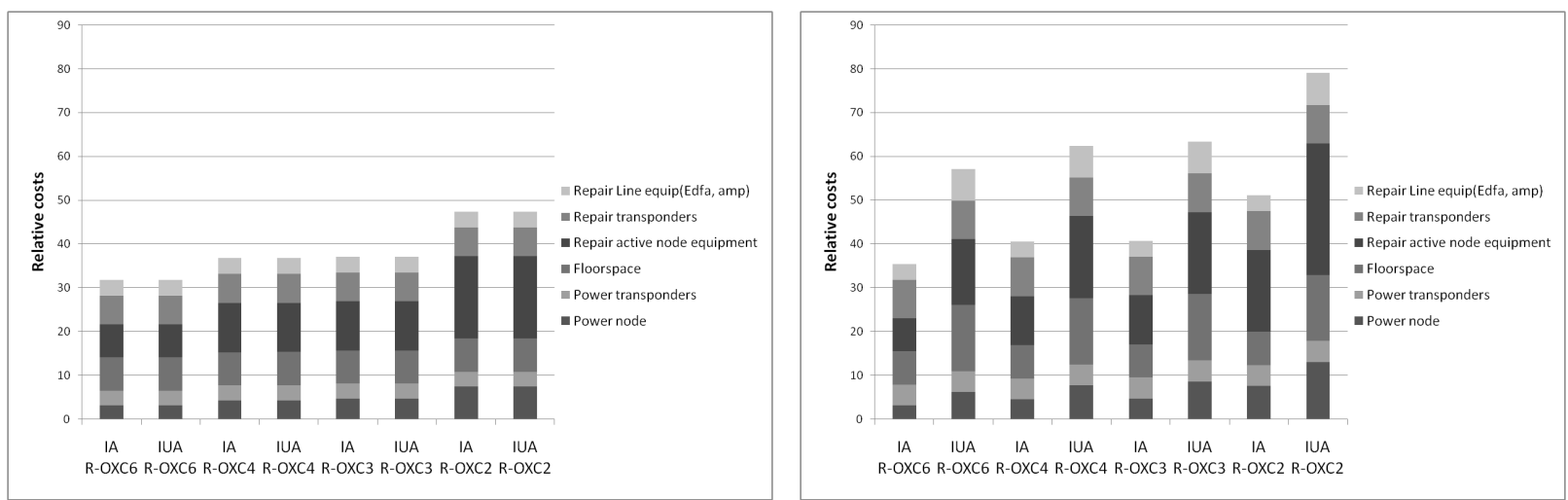

Fig. 3: OpEx of IA vs. IUA with R-OXC6 (left); OpEx of IA vs. IUA for load 1.1 (right)

and power consumption of the transponders are responsible for a slight increase for both cases when the traffic load increases (Fig. 3). Similar to the CapEx results, the sharp increase in the IUA case for loads higher than 0.8 is caused by the extra parallel line systems. Fig. 3 also depicts the computed OpEx for the four different node architectures. Overall the cost to repair the active node equipment accounts for the major part of the OpEx. The OpEx follows the same trend as the corresponding CapEx when considering the impact of the architecture and R-OXC6 incurs the lowest requirement in network-related OpEx. However, the cost of a flexible node (e.g. R-OXC2) may be counterbalanced by its superior performance under highly dynamic traffic conditions.

\section{Conclusions}

In this work we compared the resource optimization achieved by an impairment-aware network planning solution to a planning solution that is unaware of the signal QoT and showed that the optimization achieved by the former leads to capital and operational cost benefits. We also studied the role of the node architectures in the cost estimations and the results indicate that a flexible node induces higher network-related costs.

This work was supported by the EC through the FP7 projects STReP DICONET and NoE BONE.

\section{References}

1. M. Gunkel et al., "A Cost Model for the WDM Layer", in Proc. Photonics in Switching 2006.

2. A. Lord et al., "OPEX savings of all-optical core networks", in Proc. ECOC 2009.

3. R. Cardillo et al., "Considering Transmission Impairments in Wavelength Routed Networks", in Proc. OFC/NFOEC 2006, paper OFG6.

4. M. Ruffini et al., "Cost Study of Dynamically Transparent Networks", in Proc. OFC/NFOEC 2008, paper OMG2.

5. Siamak Azodolmolky et al., "An Offline Impairment Aware RWA Algorithm with Dedicated Path Protection Consideration",in Proc. OFC/NFOEC 2009, paper OWI1.

6. R. Huelsermann et al., "Cost modeling and evaluation of capital expenditures in optical multilayer networks", OSA J. of Optical Networking, vol. 7, no. 9, 2008.

7. M. De Groote et al., "Cost comparison of different Translucent Optical Network Architectures", in Proc. CTTE 2010. 